\title{
Vorwort zur elften Auflage.
}

In den dreiBig Jahren seit seinem ersten Erscheinen ist dieses Büchlein bis auf kleine Zusätze in den Erläuterungen stets in unverānderter Form wieder aufgelegt worden und hat sich in weiten Kreisen sowohl für den Schulunterricht, als für die Benutzung zu wissenschaftlichen und technischen Zwecken durchaus bewährt. Nur in einigen Punkten hatten sich im Laufe dieser langen Zeit Änderungen als wünschenswert herausgestellt, zu deren Vornahme der durch Abnutzung der bisherigen Stereotypplatten nötig gewordene neue Satz der Tafeln die günstige Gelegenheit bot. Ich habe diese Veränderungen nach reiflicher Oberlegung und nach Einholung des Rates hochgeschätzter Mathematiker und Schulmänner vorgenommen und fühle mich verpflichtet, für die grobe Bereitwilligkeit, mit welcher meine Umfrage von nah und fern beantwortet worden ist, zugleich im Namen der Herren Verleger den verbindlichsten Dank auszusprechen. Die toberzeugung, daB die von mir durchgeführten Veränderungen in der That als Verbesserungen zu betrachten sind, konnte durch die fast allseitige Zustimmung $\mathrm{zu}$ meinen Vorschlägen nur bestärkt werden; die vielfachen mir bei dieser Gelegenheit mitgeteilten Wünsche habe ich berücksichtigt, soweit dies dem Gesamtzweck des Buches $\mathrm{zu}$ entsprechen schien, und so darf ich wohl hoffen, daB die vorliegende Umarbeitung der Logarithmentafeln sich einer günstigen Aufnahme erfreuen werde. 
Diejenigen Eigenschaften, durch welche sich das Buch besonders von ähnlichen Werken unterschied, habe ich ihm zu erhalten gesucht. Die Haupttafeln, von denen sich namentlich die logarithmisch-trigonometrischen durch ihre übersichtliche Anordnung auszeichnen, haben nur insofern eine Änderung erfahren, als ich ihnen zur gröBeren Bequemlichkeit die Proportionalteile zugefügt habe, und zwar bei den trigonometrischen Tafeln für die Dezimalteilung der Minute, wie sie ja immer mehr in Gebrauch kommt. AuBer dem Vorteil einfacherer Rechnung ist die Dezimaleinteilung der Minute der Einteilung in Sekunden bei fünfstelligen Tafeln schon deshalb vorzuziehen, weil es nicht ratsam ist, ein Intervall mit selbständiger Benennung in die Rechnung einzuführen, welches so klein ist, daß man nur in den seltensten Fällen ein Rechnungsresultat mit entsprechender Genauigkeit erhält.

Auf die Dezimalteilung der Minute ist auch die zur Kreisund Winkelmessung dienende kleine Tafel III eingerichtet. Für die Logarithmen der Sinus und Tangenten kleiner Winkel sind statt der früheren Hilfstafel Formeln unter den ersten Seiten der logarithmisch-trigonometrischen Tafeln gegeben, welche eine ebenso bequeme Rechnung gestatten und den Vorteil gewähren, daB man es mit einer Tafel weniger $\mathrm{zu}$ thun hat. Die Einklammerung der zum einfachen Interpolieren ungeeigneten Differenzen wird den Anfänger vor Fehlern schützen und auch dem geübten Rechner bei schnellem Rechnen dienlich sein.

Als eine nützliche Beigabe des Werkes habe ich die abgekürzte siebenstellige Tafel beibehalten. Wenn auch der Rechner von Fach statt ihrer sich der groBen Tafeln bedienen wird, so kommt es doch namentlich beim Schulunterricht bisweilen vor, da $B$ man ohne Benutzung eines anderen Buches einen oder den anderen Wert mit gröBerer Genauigkeit zu erhalten wünscht; wenn auch vermittelst einer kleinen Nebenrechnung. Auch scheint es im pädagogischen Interesse wünschenswert, daB der Schüler sich 
gewöhnt, die Genauigkeit der Rechnung dem jedesmaligen Zwecke entsprechend einzurichten; das wird ihn vor allzu mechanischer Benutzung der Tafeln bewahren.

Dagegen habe ich die Faktorentafel und die Gaussschen Logarithmen fortgelassen, weil sie nur für besondere Zwecke hervorragenden Nutzen gewähren, während man sich meist der bekannten trigonometrischen Formeln bedient, um denselben Zweck zu erreichen, und weil es pädagogisch gewiß richtiger ist, sich mit der geringsten Zahl von Hilfsmitteln $\mathrm{zu}$ begnügen.

Die nicht logarithmische Tafel der trigonometrischen Funktionen habe ich in Intervallen von zehn zu zehn Minuten durchgeführt, während sie früher nur auf ganze Grade und auf die Minuten des ersten Grades ausgedehnt war. Diese Tafel ist für den Anfangsunterricht in der Trigonometrie von Nutzen, da sie das begrifflich Einfachere wiedergiebt, sie ist ferner häufig bequemer als die logarithmische Tafel, namentlich bei einfachen Rechnungen und wenn man nur vier bis fünf Stellen anwendet, wobei meist die gewöhnliche Interpolation gestattet ist; da die Werte siebenstellig gegeben sind, verschafft sie die Möglichkeit, für einzelne Zwecke durch ein umständlicheres Interpolationsverfahren eine größere Genauigkeit zu erreichen, als mit der logarithmisch-trigonometrischen; sie entspricht insofern der abgekürzten siebenstelligen Logarithmentafel, und bietet zugleich Gelegenheit zu einer lehrreichen Anwendung allgemeinerer Interpolationsmethoden.

Hinsichtlich der Aufstellung der Tafel bemerke ich, daB ich diejenigen Funktionswerte, welche in den VegaHülss eschen Tafeln und in den Vlacqschen Tafeln um eine Einheit der letzten Stelle differieren, mit Hilfe der Calletschen Tafeln kontrolliert habe. Dies machte eine umständliche Interpolationsrechnung nötig, da die Calletschen Tafeln zwar auf 15 Dezimalstellen genau, aber in Intervallen von einem Tausendstel des Quadranten angelegt sind. Ich fand hierbei teils die Vegaschen, teils die 
Vlacqschen Werte als die richtigeren. Namentlich fand ich auch alle diejenigen Verbesserungen gerechtfertigt, welche in den älteren Auflagen dieses Buches nach den Berechnungen von Lehmann vorgenommen waren.

Die Tafel der Quadratzahlen habe ich wegen ihrer Anwendung bei Fehlerrechnungen u. dgl. auf mehrfachen Wunsch beibehalten. Die Angaben aus der Astronomie, mathematischen Geographie und Physik, welche ich auf den letzten vier Seiten der Tafeln neu aufgenommen habe, werden gewiß vielen Lehrern willkommen sein. Hinsichtlich der astronomischen Tafeln bin ich dem Herrn Dr. Becker von der hiesigen Sternwarte, der mir bereitwilligst die den neuesten Berechnungen entsprechenden Zahlen mitgeteilt hat, $\mathrm{zu}$ besonderem Danke verpflichtet.

Eine gänzliche Umarbeitung haben die Erläuterungen erfahren. Es ist aus ihnen alles fortgelassen, was nicht unmittelbar auf die Benutzung und Einrichtung der Tafeln Bezug hat, namentlich alle Entwickelungen und Formeln aus der ebenen und sphärischen Trigonometrie. Diese Entwickelungen gehören in ein Lehrbuch, deren es jetzt viele und kürzer gefaßte giebt, als beim ersten Erscheinen der Tafeln. Die Grundformeln soll der Schüler im Kopfe haben; nur dann wird er geschickt mit ihnen operieren. Der praktische Rechner aber, der häufig kompliziertere Formeln braucht, wird selten gerade die Formeln finden, welche er haben will. Dagegen habe ich einen Punkt eingehend besprochen, der sonst meist nur sehr oberflächlich oder gar nicht behandelt wird, nāmlich die Beurteilung der erreichbaren Genauigkeit. Diese ist für ein wirkliches Verständnis der Rechnung ebensowohl von höchster Bedeutung, wie für die praktische Verwendung der Resultate, und gehört recht eigentlich in die Erläuterungen. Ich habe mich bemüht, diesen Gegenstand, über welchen vielfach unklare Vorstellungen herrschen, so darzustellen, daB ein mit den Elementen der Analysis bekannter Schüler für den wichtigsten Fall, für die einfachen Logarithmen, 
der Beweisführung vollständig folgen kann, und daB er einsehen kann, wie sich die Untersuchung auf alle durch Potenzreihen darstellbaren Funktionen ausdehnen läßt.

Die Anordnung der Tabellen habe ich so getroffen, da $\beta$ die am häufigsten gebrauchten voranstehen, nämlich die fünfstelligen logarithmischen und trigonometrischen.

\section{Vorwort zar dreiundzwanzigsten Auflage.}

Seit der vierzehnten Auflage sind auf S. 36 einige Werte nach Angabe des Herrn Professor Mehler in Elbing und nach den Vorschlägen des Herrn Geheimrat Professor För ster in Berlin und des Herrn Professor Becker in Gotha die astronomischen Tabellen verbessert. In der neunzehnten ist im AnschluB an die gesetzliche Einführung der mitteleuropäischen Zeit eine Änderung und Erweiterung der Ortstafel vorgenommen worden.

Die jetzt vorliegende Auflage enthält eine Verbesserung der Tabelle V nach den Vorschlägen des Herrn P. Winnertz in Krefeld. Die Zahl der in die Abteilung A aufgenommenen Mantiffen konnte nämlich beträchtlich verkleinert werden, ohne den Zweck dieser Abteilung zu beeinträchtigen. Hierdurch ist es möglich geworden, die Mantiffen in A in ebenso grober Schrift zu setzen, wie die in B, was für das Auge eine große Erleichterung ist. Gleichzeitig sind, um die erreichbare Genauigkeit zu vermehren, jene Mantiffen acht. stellig gegeben. Im Zusammenhange mit diesen Verbesserungen wurde in den Erläuterungen eine Änderung der $\S \S 10-13$ erforderlich.

Berlin, im Januar 1900.

Der Herausgeber. 